Published as: Fountas N.A., Živković S., Benhadj-Djilali R., Stergiou C.I., Majstorovic V.D., Vaxevanidis N.M. (2017) Intelligent Dual Curve-Driven Tool Path Optimization and Virtual CMM Inspection for Sculptured Surface CNC Machining. In: Majstorovic V., Jakovljevic Z. (eds) Proceedings of 5th International Conference on Advanced Manufacturing Engineering and Technologies. NEWTECH 2017. Lecture Notes in Mechanical Engineering. Springer, Cham, pp.345-356. ISBN: 9783319564296, ISSN: 2195-4356.

https://doi.org/10.1007/978-3-319-56430-2_25 


\title{
INTELLIGENT DUAL CURVE-DRIVEN TOOL PATH OPTIMIZATION AND VIRTUAL CMM INSPECTION FOR SCULPTURED SURFACE CNC MACHINING
}

\author{
N.A. Fountas ${ }^{1,4}$, S. Živković ${ }^{2}$, T.V. Sibalija ${ }^{3}$, R. Benhadj-Djilali ${ }^{4}$, C.I. Stergiou ${ }^{5}$, V.D. Majstorovic ${ }^{6}$, \\ N.M. Vaxevanidis ${ }^{1^{*}}$
}

${ }^{1}$ Laboratory of Manufacturing Processes and Machine Tools (LMProMaT), Department of Mechanical Engineering Educators, School of Pedagogical and Technological Education (ASPETE), ASPETE Campus, N. Heraklion, GR 14121 Athens, Greece

${ }^{2}$ Military Technical Institute Belgrade, Coordinate Metrology Lab, Ratka Resanovića 1, 11030 Belgrade, Serbia.

${ }^{3}$ Faculty of Management, Faculty of Information Technology, Metropolitan University, Tadeusa Koscuska 63, 11000 Belgrade, Serbia

${ }^{4}$ Faculty of Science, Engineering and Computing, Kingston University, Roehampton Vale Campus, Friars Avenue, Kingston upon Thames, London SW15 3DW, UK

${ }^{5}$ Department of Mechanical Engineering, Piraeus University of Applied Sciences, GR 12244 Egaleo, Greece

${ }^{6}$ Faculty of Mechanical Engineering, University of Belgrade, Kraljice Marije 16, 11120 Belgrade, Serbia

* corresponding author; vaxev@aspete.gr

\begin{abstract}
This paper investigates the profitability of a dual-curve driven surface finish tool path under the concept of optimizing crucial machining parameters such as toroidal end-mill diameter, lead angle and tilt angle. Surface machining error as well as tool path time are treated as optimization objectives under a multi-criteria sense, whilst a central composite design is conducted to obtain experimental outputs for examination and, finally, fit a full quadratic model considered as the fitness function for process optimization by means of a genetic algorithm. A benchmark sculptured surface given as a second-order parametric equation was tested and simulated using a cutting-edge manufacturing modeling software and best parameters recommended by the genetic algorithm were implemented for validation. Further assessment involves the virtual inspection to selected profile sections on the part. It was shown that the approach can produce dual-curve driven tool trajectories capable of eliminating sharp scallop heights, maximizing machining strip widths as well as maintaining smoothness quality and machining efficiency.
\end{abstract}

\section{Keywords}

Dual-curve driven tool paths; toroidal end-mills; multi-criteria optimization; sculptured surface machining; genetic algorithms 


\section{Introduction}

A large number of multi-axis surface finish tool paths are now available to machine sculptured surfaces such that reduced cycle times as well as high quality criteria are met. Multi-axis surface finish tool paths are accompanied with a number of machining parameters ought to be determined with special emphasis to inclination angles for the two rotational axes found in 5-axis CNC machining. Even though a resemblance exists among various swept-cut style tool paths in terms of parameter specification through the manufacturing software interface, the controllability of the trajectory flow may be different and remains unknown to the public [1]. Such tool paths available to commercially available CAM software systems are iso-scallop [2], iso-planar [3] as well as isoparametric [4].

To ensure consistency in cutting performance and capture profitability, experimental investigations ought to be conducted using a given tool path to be applied on a free-form surface, prior to its implementation. To that extend, several researchers have examined a variety of tool paths for complex surfaces in order to provide insight concerning their settings aiming at optimizing criteria such as scallop height, tool path length, surface deviation using the theoretical CAD model as a reference, etc. [5], as well as physical machinability indicators such as roughness [6]. Related research concerning experimental investigations for tool path strategies also involves the optimal formulation of various tool paths using artificial techniques. Ulker et al., [7] implemented an immune approach to produce properly spaced cutter locations given a cut tolerance, for both $u$ and $v$ directions referring to the 3D parametric space. Agrawal et al., [8] implemented a genetic algorithm to formulate master cutter paths that will smoothly unite the tool trajectories of different limiting contours.

The cutting tool type is the most important factor to which corresponded surface finish tool path parameters are refer to. Hence, machining surface creation is largely depended on the tool type and its contact to that surface [9]. In recent years toroidal as well as barrel type end mills have been attracting much attention whilst a large number of research works report their benefits against other tool types like ball end mills or flat-end mills. Benefits of torus end mills include larger effective radii towards path trajectories [10], gouge avoidance owing to the absence of sharp edges when machining with normal to surface, as well as attaining larger machining strip widths [11]. Hendriko in [12] developed an extended analytical boundary method to determine the machining geometry during free-form milling. The algorithm was developed by considering inclination angles. The proposed method was successfully implemented to calculate the scallop for two model parts with different surface profiles. The accuracy was verified by comparing the scallop height calculated using the proposed method with those measured using a cutting-edge machining simulation environment. For these experiments, a toroidal cutter was applied. Investigation for the usage of toroidal end-mills is mainly conducted for tool path planning in terms of tool positioning such that a curvature matching between the tool's geometry and the given sculptured surface exists in every cutter contact point. This assumption spans a noticeable number of research works for tool positioning, such as those presented in Roman et al., [13] dealing with the "rolling-ball" method applied to 31/21/2-axis machining, as well as in Warkentin et al., [14] where a number of discrete tool positioning methods for 5-axis surface tool paths are assessed using a toroidal cutter.

The main thrust of this paper is to examine a particular multi-axis surface finish tool path based on two drive curves for its cutting style definition, aiming at attaining the lowest surface deviation and the lowest machining time. Note that values for machining parameters ought to produce a tool path under the preset cut tolerance for the successful interpolation. First, the tool path's control is described whilst a number of machining experiments are established using a benchmark sculptured surface, to obtain results for machining deviation error and tool path time. From these experiments a fitness model under the principles of least squares approach is developed and it is utilized as the objective function for a genetic algorithm, to sequentially evaluate it so as to arrive at optimal solutions for machining parameters; cutting tool diameter as well as 5-axis inclination angles (lead and tilt). The experiments are conducted and analyzed using a machining simulation environment [15]. 


\section{Intelligent tool path parameter selection for dual-curve driven trajectories}

\section{Dual-curve driven multi-axis surface tool paths}

According to the philosophy underpinning the control of dual-curve driven tool surface paths, the largest possible machining strip widths can be attained by positioning the cutting tool given its geometry, radial cut engagement (stepover), and tool inclination angles. The first drive-curve is responsible for indicating a set of cutter contact points for the formulation of the primary cutting path. The secondary cutting path comes to be as the outcome of the first drive curve with the narrowest strip width among the above maximum machining strip width at each cutter contact point. Thereby, the tool path strategy repositions the tool utilizing the pair of cutter contact points along the two drive curves, resulting in uniformly planned trajectories, free of gouging and large surface deviation error (Fig. 1). By taking into account Fig. 1, for a given surface $S$, the primary drive-curve $d c_{i}{ }^{1}$ is discretized to $C C_{i, j}$ sampling cutter contact points, with $C C_{i, j}=(j=1,2, \ldots, n)$, considering the cut tolerance and the discretization algorithm of CAM software for the strategy. For every $c c_{i, j}$ point on $d c_{i}{ }^{1}$ the corresponding cutter contact point $C C_{i+1, j}$ may be obtained, as well as the related machining strip width $m s w_{i, j}$ at $C C_{i, j}$. In other words, $m s w_{i, j}$ is directly related to the path interval or the step over distance of $S$. The next drivecurve $d c_{i+1}{ }^{1}$ is the result of $d c_{i}^{1}$ and $m s w_{i, j}$ whereas the curve linking $c c_{i, j}$ and $c c_{i+1, j}$ is finally the secondary drive-curve $d c_{i}^{2}$ for the tool path.

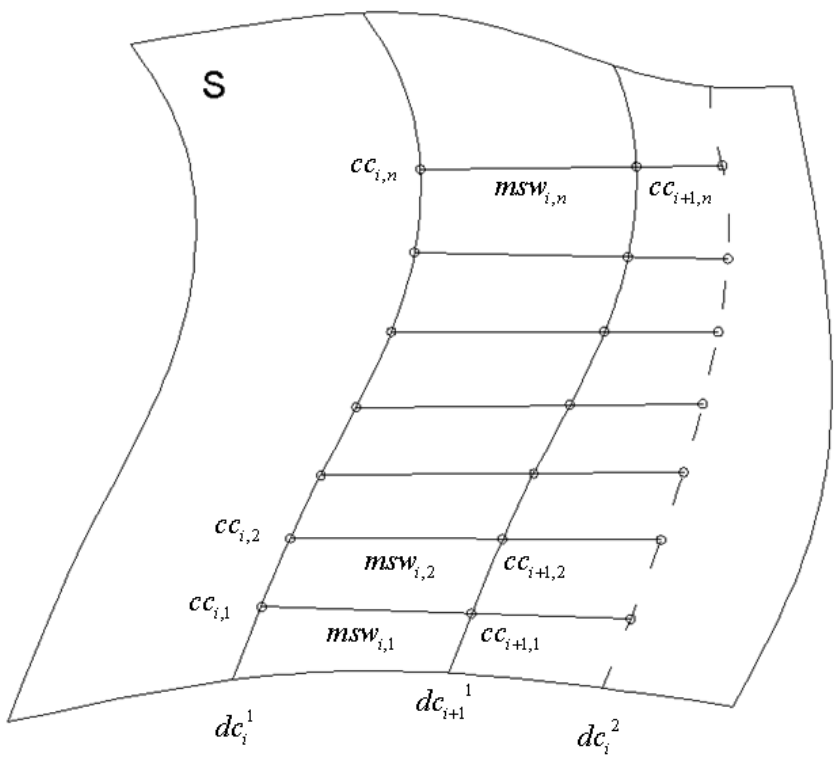

Fig.1: General representation of a dual-curve driven tool path mechanism.

An important function of this tool path control is also the sequential alternation between primary and secondary drive curves that leads to scallop elimination between subsequent step over passes, smoothing this way the adjacent regions. Further details on this particular tool path generation as well as new developments on the basis of dual-driven 5-axis surface CNC planning can be found in [16].

\section{Experimental results}

The sculptured surface selected for the analysis was a benchmark $S(u, v)$ second-order parametric surface described by the following equations. The surface was of an open-form contour, commonly found in mold/die industry and it has been used by many researchers $[16,17]$. 
$S(u, v)=\left[\begin{array}{c}-94.4+88.9 v+5.6 v^{2} \\ -131.3 u+28.1 u^{2} \\ a_{1}+a_{2}\end{array}\right]$

with,

$a_{1}=5.9\left(u^{2} v^{2}+u^{2} v\right)-3.9 v^{2} u+76.2 u^{2}$ and $a_{2}=6.7 v^{2}-27.3 u v-50.8 u+25 v+12.1$.

Fig.2a shows the CAD model of the benchmark sculptured surface modeled using the above equations; whilst Fig. $2 \mathrm{~b}$ depicts the dual-curve driven surface finish tool path that was investigated.

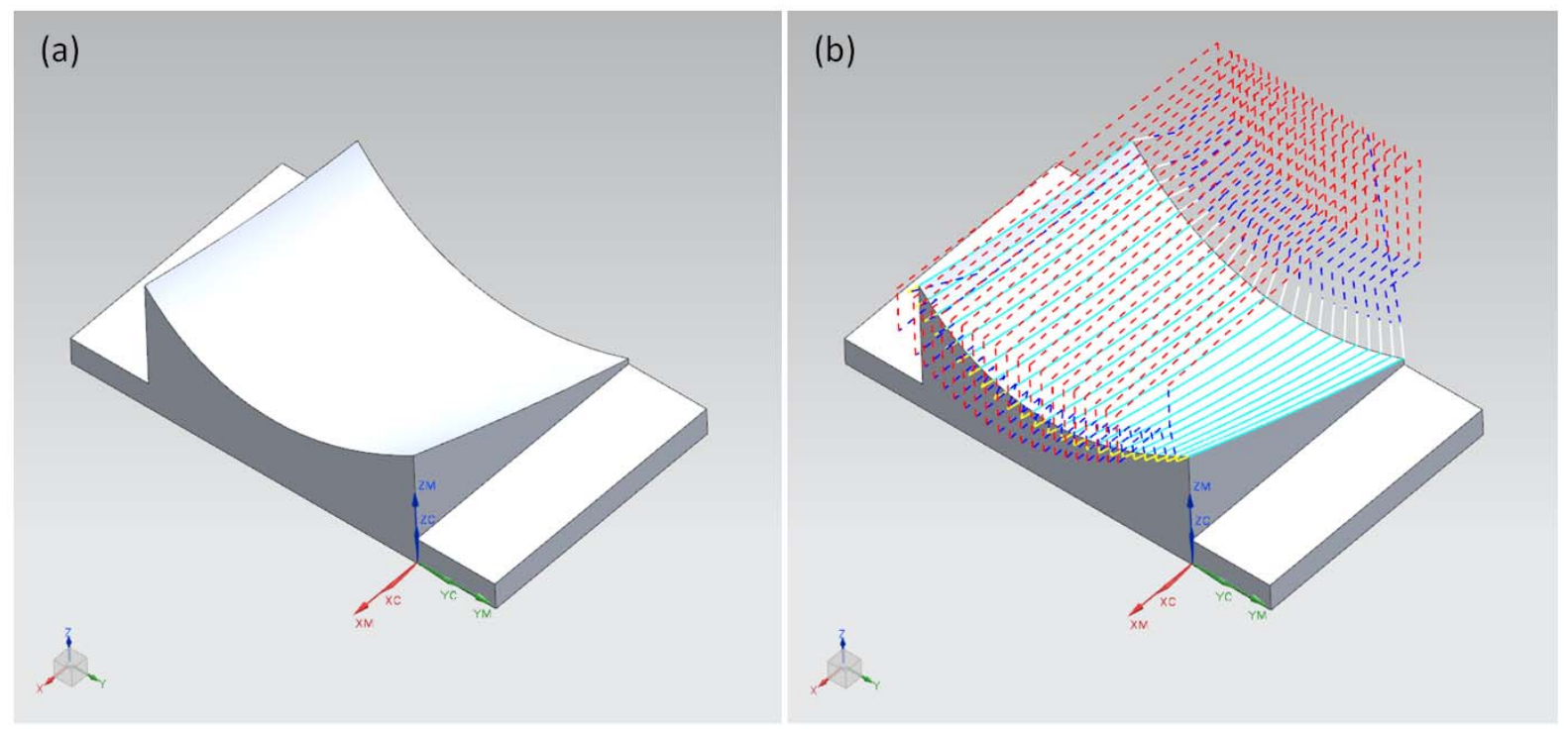

Fig. 2: Benchmark parametric surface: (a) 3D CAD benchmark model; (b) dual-curve driven surface finish tool path for machining experiments.

\section{Fitness function formulation and genetic algorithm parameters}

The proposed tool path optimization scheme suggests the implementation of a genetic algorithm as an efficient heuristic technique capable of converging to an optimum solution for the objectives involved to the problem. Prior to its involvement the search space representing the problem's domain should be formulated via the fitness function generation. Aiming at building a robust fitness model for the algorithmic evaluations, a number of machining simulation experiments was conducted adopting the central composite design under the response surface methodology (RSM) having two continuous factors (the two inclination angles), one categorical factor (the cutting tool diameter), and two equally weighted responses referring to the machining error and tool path time.

Experiments were simulated in Siemens ${ }^{\circledR}$ NX-11 CAM environment using the aforementioned tool path strategy. Spindle speed was set to $16000 \mathrm{rpm}$ whilst feed rate was set to $5000 \mathrm{~mm} / \mathrm{min}$. Cut tolerance was set to $0.1 \mathrm{~mm}$; large enough so as to obtain noticeable variations that would ease examination efforts. Step over distance was controlled through scallop height equal to cut tolerance; $0.1 \mathrm{~mm}$ for the same reasons. Hence different adjacent regions were examined according the cutting diameter. All three toroidal cutters simulated were of corner radius equal to $3 \mathrm{~mm}$. The combination of the independent parameters and their three 'low-middle-high' levels gave a total of 21 experiments; 7 for each torus-type cutting tool diameter ( $8 \mathrm{~mm} ; 12 \mathrm{~mm}$ and $16 \mathrm{~mm})$. The levels for the two inclination angles were 0, 22.5 and 45 degrees, and -20, 0, 20 degrees for the three levels of lead and tilt angles, respectively. For each test, series of analysis points were obtained via normal-to-surface measurements and finally compute the average value representing the surface deviation objective. Machining time was directly obtained in the end of each experimental test. Results for the two objectives were statistically analyzed to study the correlation possibility by fitting the data via three sets of full quadratic models, one per cutting tool diameter and one per objective. The models considered for the fitness of algorithmic evaluations were 
those generated for $16 \mathrm{~mm}$ diameter toroidal cutting tool, since it was found the most beneficial in terms of the multi-criteria optimization between surface deviation and machining time. The final model taken as the fitness was the root of the squared sum of the two objectives after their normalization procedure to remove the inherent bias owing to different magnitudes. Table 1 shows the parameters, their levels as well as the experimental results from the machining simulations.

Table 1. Design of experiments and results for objectives.

\begin{tabular}{|c|c|c|c|c|c|c|c|c|}
\hline Run & $\begin{array}{l}\text { Lead } \\
\text { (deg) }\end{array}$ & $\begin{array}{l}\text { Tilt } \\
\text { (deg) }\end{array}$ & $\begin{array}{l}\text { TorusRc3 } \\
(\mathrm{mm})\end{array}$ & $\begin{array}{l}\text { SurfDev } \\
\text { (mm) }\end{array}$ & $\begin{array}{l}\text { TlpTime } \\
\text { (min) }\end{array}$ & SurfDevNorm & TlpTimeNorm & Pareto \\
\hline 1 & 0 & -20 & 8 & 0.18624 & 53.20 & 0.332951945 & 0.996254682 & 53.2 \\
\hline 2 & 45 & -20 & 8 & 0.062233 & 44.20 & 0.111257509 & 0.827715356 & 44.2 \\
\hline 3 & 0 & 20 & 8 & 0.179627 & 53.19 & 0.321129505 & 0.996067416 & 53.19 \\
\hline 4 & 45 & 20 & 8 & 0.041593 & 44.15 & 0.074358195 & 0.826779026 & 44.15 \\
\hline 5 & 22.5 & 0 & 8 & 0.1211 & 38.13 & 0.216497426 & 0.714044944 & 38.13 \\
\hline 6 & 22.5 & 0 & 8 & 0.1211 & 38.13 & 0.216497426 & 0.714044944 & 38.13 \\
\hline 7 & 22.5 & 0 & 8 & 0.1211 & 38.13 & 0.216497426 & 0.714044944 & 38.13 \\
\hline 8 & 0 & -20 & 12 & 0.55936 & 53.25 & 1 & 0.997191011 & 53.253 \\
\hline 9 & 45 & -20 & 12 & 0.278307 & 35.06 & 0.497545409 & 0.656554307 & 35.061 \\
\hline 10 & 0 & 20 & 12 & 0.55216 & 53.20 & 0.987128146 & 0.996254682 & 53.203 \\
\hline 11 & 45 & 20 & 12 & 0.739373 & 34.47 & 1.32181958 & 0.645505618 & 34.478 \\
\hline 12 & 22.5 & 0 & 12 & 0.11824 & 27.01 & 0.211384439 & 0.505805243 & 27.01 \\
\hline 13 & 22.5 & 0 & 12 & 0.11824 & 27.01 & 0.211384439 & 0.505805243 & 27.01 \\
\hline 14 & 22.5 & 0 & 12 & 0.11824 & 27.01 & 0.211384439 & 0.505805243 & 27.01 \\
\hline 15 & 0 & -20 & 16 & 0.153747 & 53.40 & 0.274862343 & 1 & 53.4 \\
\hline 16 & 45 & -20 & 16 & 0.067887 & 30.04 & 0.121365489 & 0.562546816 & 30.04 \\
\hline 17 & 0 & 20 & 16 & 0.195833 & 53.31 & 0.350101902 & 0.998314607 & 53.31 \\
\hline 18 & 45 & 20 & 16 & 0.320147 & 29.32 & 0.57234518 & 0.54906367 & 29.322 \\
\hline 19 & 22.5 & 0 & 16 & 0.16306 & 21.31 & 0.291511728 & 0.39906367 & 21.311 \\
\hline 20 & 22.5 & 0 & 16 & 0.16306 & 21.31 & 0.291511728 & 0.39906367 & 21.311 \\
\hline 21 & 22.5 & 0 & 16 & 0.16306 & 21.31 & 0.291511728 & 0.39906367 & 21.311 \\
\hline
\end{tabular}

Optimization for simultaneously minimizing the criteria is subjected to the specified ranges of machining parameters. The ranges are determined considering the lower and upper levels of the experimental design presented above. When dealing with engineering optimization problems it is a common technique that heuristics will run several times under a trial-and-error experimentation so as to find the optimal parameter setting referring to intelligent operators. This should be done given the fact that no certain recommendations for best combinations among intelligent parameter settings exists. By employing the genetic algorithm to optimize the fitness function, ten sequential runs were executed using the Mathworks Matlab ${ }^{\circledR}$ R2016a optimization toolbox. The parameters for the genetic algorithm implemented to optimize the objective functions were as follows:

\footnotetext{
- $\quad$ Population size: 50

- $\quad$ Generations: 60

- $\quad$ Scaling function: Rank

- Selection scheme: Stochastic uniform

- $\quad$ Elite count: $0.05 *$ population size $=2$

- Crossover type: Single-point crossover

- $\quad$ Crossover fraction: 0.8
}

The population type was encoded as double vector and the range for the search was (low:[0, -20], high:[45, 20]). Ranking was applied for scaling fitness values so as to select parents for next generations. Selection assigns higher probability for reproduction to better fitted individuals whose fitness scores are ranked accordingly. 
Ranking is achieved by considering all fitness scores attained by candidate solutions. Individuals selected for reproduction followed the stochastic uniform scheme where each parent corresponds to a sub-string of the chromosome's binary string of length proportional to its scaled value. According to this scheme, the algorithm allocates a parent from the sub-string through its equidistant movement towards the whole chromosome string. The default value determining the elite count for individuals was 0.05 times the population size which in this case is equal to 50 . Hence 2 elite individuals are guaranteed to survive to the next generation.

Crossover type was a single-point. Crossover fraction determines the number of individuals other than elite ones which are produced through crossover operator. Remaining individuals are produced owing to mutation. Since optimization for parameters is restricted to lower and upper ranges mentioned, a constraint dependent mutation function was applied to the module. The outputs selected for evaluation were the best fitness value attained the best mean fitness and the best individual (final point). This implies that the optimum result obtained by the genetic algorithm corresponding to the Pareto combined criterion for the tool path is expected to be lower than, or at least equal to the minimum Pareto value attained for the experimental results. Mean fitness values of final points the algorithm attained were also assessed and compared against the average of the experimental results as well as the average of predicted ones by applying the full quadratic model.

The correlation coefficient $\mathrm{R}^{2}$ for the final multi-criteria Pareto objective implemented as the fitness function was found equal to $82.22 \%$. The optimal machining parameters recommended were the average values from ten series of evaluations whilst they checked whether they fall in the same range as the one restricting the values for parameters selection from the experimental design shown in Table 1. Fig. 3 shows the correlation among the normalized experimental multi-criteria Pareto results versus the one corresponding to the prediction capability of the full quadratic multi-criteria Pareto objective. The convergence diagram of the best fitness score obtained is depicted in Fig. 4. The resulting fitness score is by far lowest than the lowest experimental result for Pareto objective (21.311) as well as the lowest one predicted by the regression equation (23.764). To quantitatively illustrate the benefits of the optimization approach, the best value representing the fitness score was expressed as a percentage of the aforementioned lowest experimental results. By taking into account the lowest experimental result, the genetic algorithm reduced the mean for the Pareto objective by $78.28 \%$, whereas considering the lowest predicted result when employing the regression model the gain is $80.52 \%$ reduction of the corresponding mean.

Experimental vs predicted results for multi-criteria Pareto objective

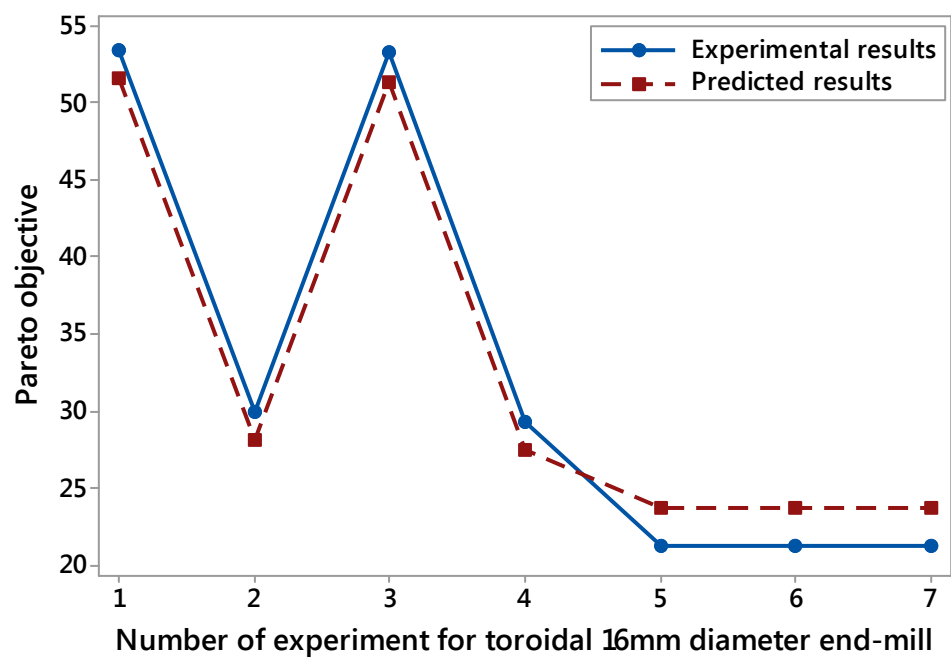

Fig. 3: Experimental versus predicted results for multi-criteria Pareto objective. 


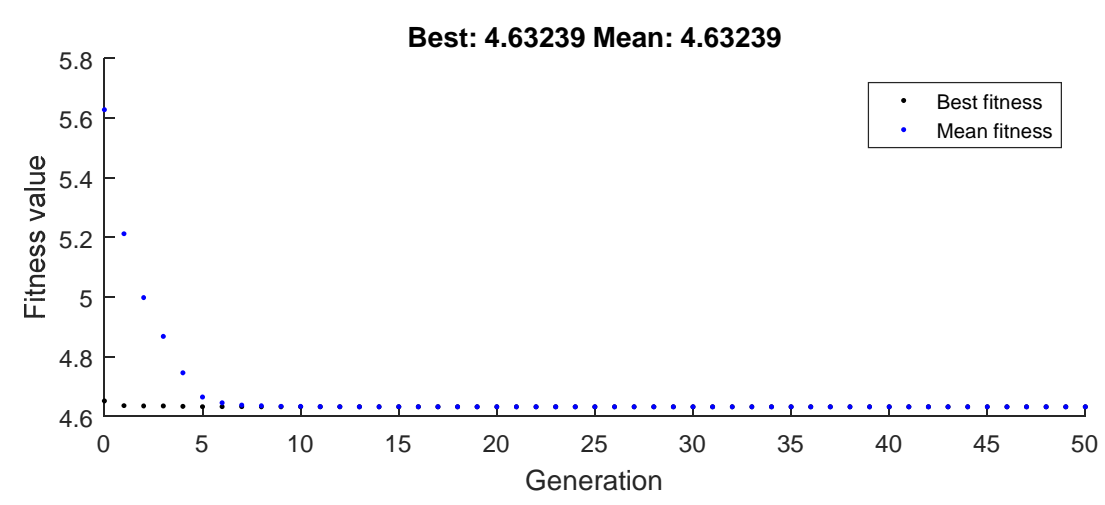

Fig. 4: Fitness function evaluations.

\section{Simulation results}

Optimal settings for machining parameters recommended by the genetic algorithm were passed onto Siemens ${ }^{\circledast}$ NX 11 CAM environment to validate the proposed experimental investigation and optimization procedure for this particular tool path. These parameters were: $\varnothing 16 \mathrm{Rc} 3$ toroidal end-mill; Lead angle $=30.857$ degrees and Tilt angle $=19.99$ degrees. Fig. 5 shows the CAM simulation outputs of the lowest experimental result and the optimum one using the genetic algorithm. Fig. 5 a refers to the output obtained when using a lead angle equal to 22.5 degrees and tilt angle equal to 0 degrees under the $\varnothing 16 \mathrm{Rc} 3$ toroidal end-mill. Fig. $5 \mathrm{~b}$ refers to the CAM simulation output when employing the parameters recommended by the genetic algorithm for the process. The former tool path came with a total machining time equal to $28 \mathrm{~min}$ and $37 \mathrm{sec}$, whereas the latter tool path came with a total machining time equal to $21 \mathrm{~min}$ and $31 \mathrm{sec}$. By taking into account the machining strip widths remained as excess material on the part's free form surface, it can be seen that they are more profound in the case of employing parameters from the lowest experimental result's combination than those resulted in the optimized case.
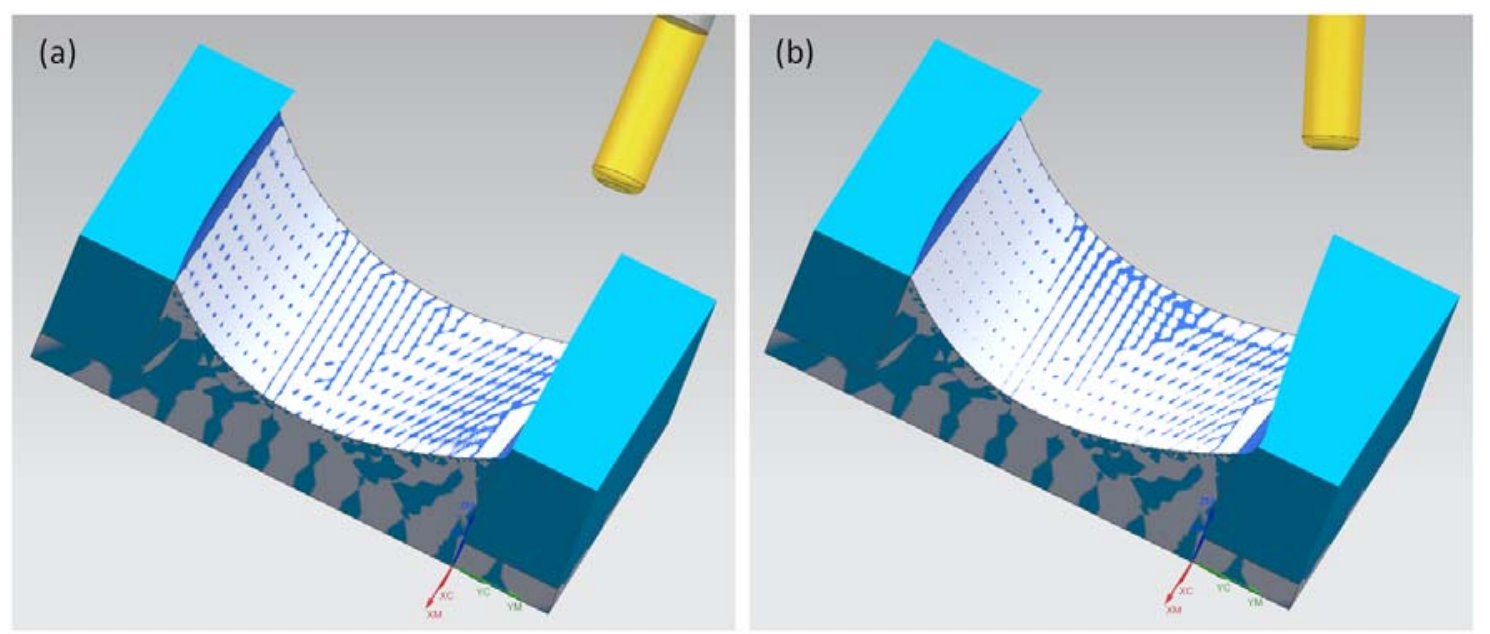

Fig. 5: (a) CAM simulation output using the lowest experimental result parameters; (b) CAM simulation output using the parameters recommended by the genetic algorithm.

\section{Virtual CMM inspection results}

Further validation of the experimental results involves the virtual CMM inspection. For the benchmark part machined using the optimal parameters for the dual-curve driven surface finish tool path, a series of measurements were performed in Decartes coordinate system XYZ [18]. Fig.6 shows details of all steps performed in the virtual inspection. 


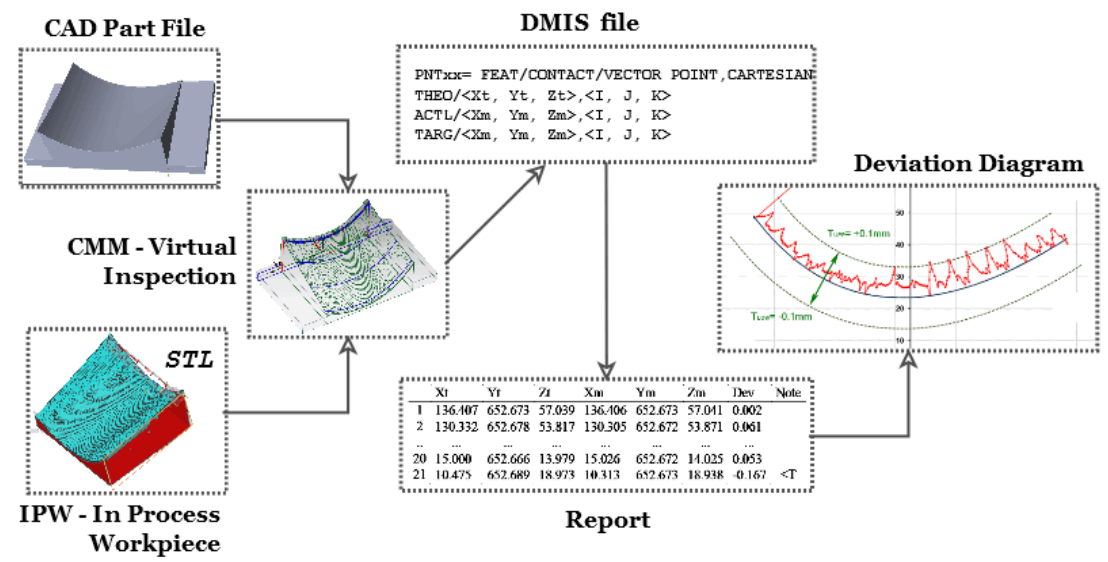

Fig. 6: Virtual inspection steps

Measured values were verified using the $[+0.1 \mathrm{~mm}-0.1 \mathrm{~mm}]$ tolerance field. Fig. 7 shows the surface deviation error between the virtually machined surface and the curved profile of the design surface in four selected sections. The selected sections are referred to the OYZ plane at $X=-5 \mathrm{~mm}, X$ $=-30 \mathrm{~mm}, X=-60 \mathrm{~mm}$, and $X=-90 \mathrm{~mm}$ respectively. One may notice that the error distribution in all three resulting profiles falls within the specified tolerance limits, whereas no undercuts or gauges are detected. Moreover it is evident that most of the red dots illustrating the surface machining error vary from [0+0.05] or even less than $+0.05 \mathrm{~mm}$ of tolerance as the $Y$ coordinates increase. Regions to where large scallops are to be experienced are the ones distributed among the adjacent tool path trajectories where no sharp edges were finally tracked. Machining strip widths are also found continuous and smooth whilst the peaks in the figure suggest large; yet rounded scallops that join these uniform machining strip widths.

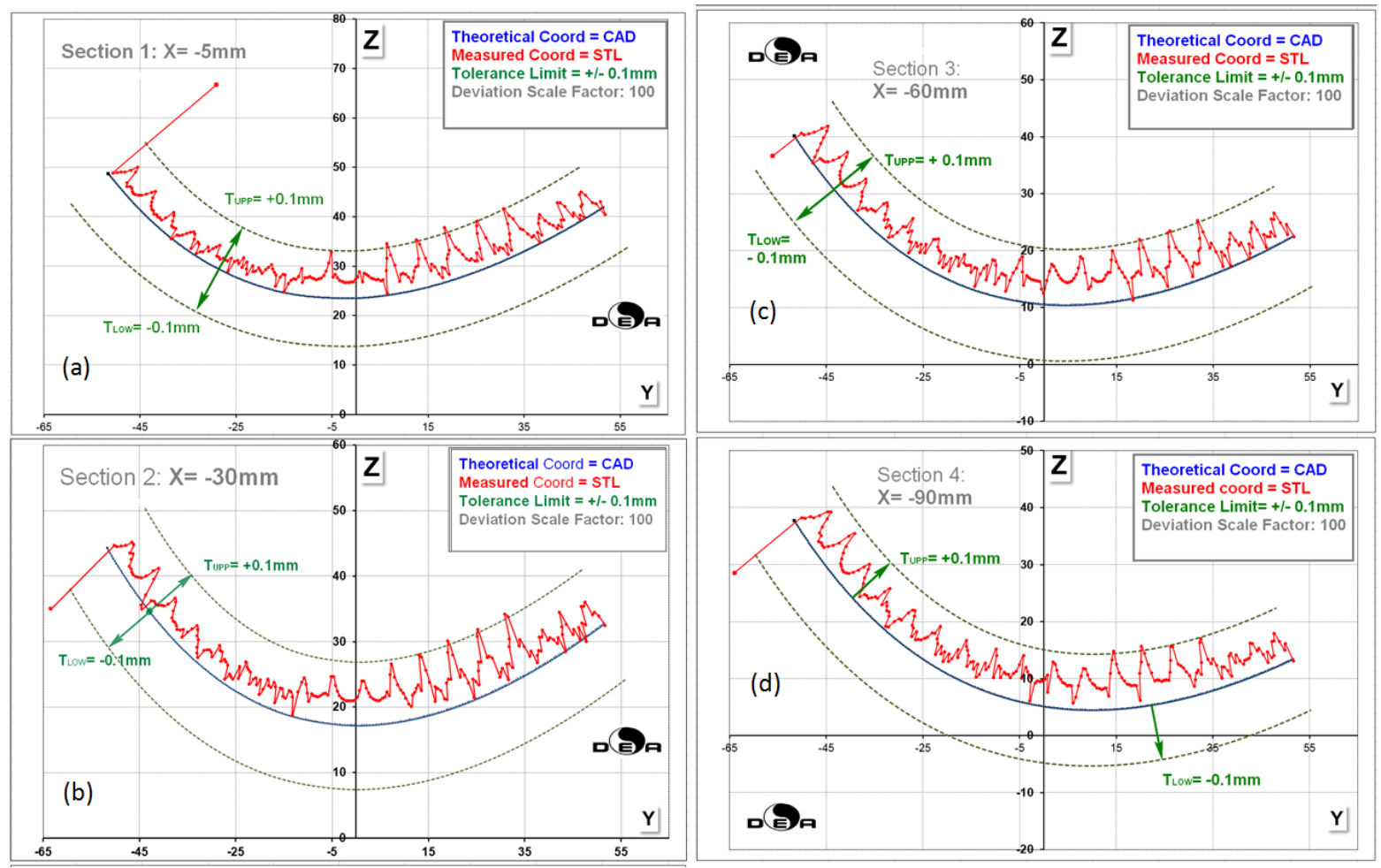

Fig. 7: Measured results of the machining-simulated surface: (a) section $X=-5 \mathrm{~mm}$; (b) section $X=-30 \mathrm{~mm}$; (c) section $X=-60 \mathrm{~mm}$; (d) section $X=-90 \mathrm{~mm}$. 
The developed methodology was successfully tested on a real test part. Fig. 8 shows an actual coordinate inspection of the manufactured test part.

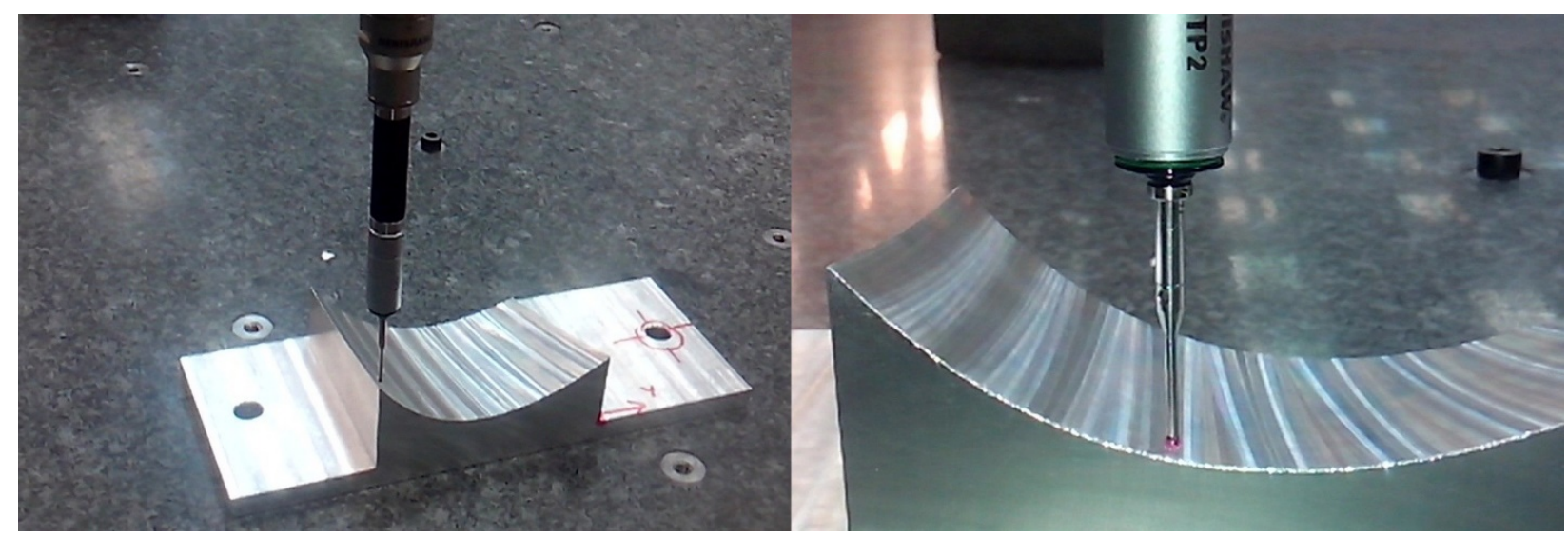

Fig. 8: Coordinate inspection of the manufactured test part

\section{Conclusions}

A particular multi-axis surface finish tool path, based on two drive curves for its cutting style definition, was virtually examined. Its efficiency was decided upon the context of finding optimal parameter values for the two inclination angles lead and tilt, whilst machining by employing toroidal end-mills. To characterize the performance of such a tool path, a genetic algorithm was implemented undertaking the multi-criteria minimization between surface machining error and tool path time. A regression model built through an experimental design played the role of a fitness function for the genetic algorithm. Despite the frequently mentioned shortcomings in terms of generalization, the regression model was capable of describing the problem's domain such that first-order continuous profiles could be generated on the tested sculptured surface. Looking further ahead, more tool paths for multi-axis surface finish will be examined so as to enhance shops' practices implementing advanced manufacturing software for sculptured surface CNC machining.

\section{References}

[1] Zhiwei Lin \& Jianzhong Fu \& Hongyao Shen \& Wenfeng Gan, An accurate surface error optimization for fiveaxis machining of freeform surfaces, Int J Adv Manuf Technol (2014) 71:1175-1185.

[2] Suresh K, Yang D C H. Constant scallop-height machining of free-form surfaces. Journal of Engineering for Industry 1994; 116(2): 253-259.

[3] S. Ding, M.A. Mannan, A.N. Poo, D.C.H. Yang, Z. Han. The implementation of adaptive isoplanar tool path generation for the machining of free-form surfaces, Int. J. Adv. Manuf. Technol. 26 (7) (2005)852-860.

[4] Subhajit Sarkar and Partha P Dey A new iso-parametric machining algorithm for free-form surface. Proc IMechE Part E: J Process Mechanical Engineering 2014, Vol. 228(3) 197-209.

[5] X. F. Zhang, J. Xie, H. F. Xie, L. H. Li. Experimental investigation on various tool path strategies influencing surface quality and form accuracy of CNC milled complex freeform surface, Int. J. Adv. Manuf. Technol. 59 (2012) 647-654.

[6] A.F. De Souza, A. Machado, S.F. Beckert, A.E. Diniz. Evaluating the roughness according to the tool path strategy when milling free form surfaces for mold application, Proc. CIRP 14 (2014) 188-193.

[7] E. Ülker, M. E. Turanalp,H. S. Halkaci, An artificial immune system approach to CNC tool path generation, Journal of Intelligent Manufacturing, 2009, 20(1), 67-77.

[8] R.K. Agrawal, D.K. Pratihar, A.R. Choudhury, Optimization of CNC isoscallop free form surface machining using a genetic algorithm. Int. J. Mach. Tools Manuf. 46(7-8), 2006, 811-819.

[9] S. Bedi, F. Ismail, M. J. Mahjoob,Y. Chen. Toroidal versus ball nose and flat bottom end mills. Int. J. Adv. Manuf. Technol. 1997, 13(5), 326-332.

[10] J.-M. Redonnet, S. Djebali, S. Segonds, J. Senatore, W. Rubio, Study of the effective cutter radius for end milling of free-form surfaces using a torus milling cutter, Computer-Aided Design, 45(6), 2013, 951-962.

[11] Lee Y S. Non-isoparametric tool path planning by machining strip evaluation for 5-axis sculptured surface machining. Computer-Aided Design 1998; 30(7): 559-570.

[12] H. Hendriko, "Analytical Boundary Method for Obtaining Feed Scallop of Toroidal Cutter in Multi-Axis Milling", Key Engineering Materials, Vol. 728, pp. 48-53, 2017. 
[13] A. Roman, E. Barocio, J.C. Huegel, S. Bedi, Rolling ball method applied to $3 \frac{1}{2} \frac{1}{2}$-axis machining for tool orientation and positioning and path planning, Advances in Mechanical Engineering 2015, 7(12) 1-12.

[14] A. Warkentin, F. Ismail, S. Bedi, Comparison between multi-point and other 5-axis tool positioning strategies, Int. J. Mach. Tools Manuf. 40 (2000) 185-208.

[15] L.T. Tunç, O.M. Ozkirimli, E. Budak. Machining strategy development and parameter selection in 5-axis milling based on process simulations, Int. J. Adv. Manuf. Technol. 85 (2016) 1483-1500.

[16] Rufeng X., Zhitong C., Wuyi C., Xianzhen W., Jianjun Z. Dual Drive Curve Tool Path Planning Method for 5-axis NC Machining of Sculptured Surfaces. Chinese Journal of Aeronautics, (2010), 23, 486-494.

[17] Warkentin A, Ismail F, Bedi S. Multi-point tool positioning strategy for 5-axis machining of sculptured surfaces. Computer Aided Geometric Design 2000; 17(1): 83-100.

[18] Sibalija T.V., Zivkovic S.P., Fountas N.A., Majstorovic V.D., Macuzic J. Z., Vaxevanidis N.M. Virtual Optimisation of CAI Process Parameters for the Sculptured Surface Inspection, Procedia CIRP 57, 2016, 574-579. 